\title{
Entrapping of a vortex pair interacting with a fixed point vortex revisited. Part II:
}

\section{Finite size vortices and the effect of deformation}

\author{
Jean N. Reinaud,, a) Konstantin V. Koshel, ${ }^{2, \text { b) }}$ and Eugene A. Ryzhov, 2, c)
}

1) Mathematical Institute, University of St Andrews, North Haugh, St Andrews KY169SS, United Kingdom

2) V.I.Il'ichev Pacific Oceanological Institute of FEB RAS, 43, Baltiyskaya Street, Vladivostok, 690041, Russia

3) Department of Mathematics, Imperial College London, London SW' 2AZ, United Kingdom

We investigate the evolution of a pair of two-dimensional, opposite-signed, finitesize vortices interacting with a fixed point vortex. The present paper builds on the accompanying study [Koshel, K. V., Reinaud, J. N., Riccardi, G. and Ryzhov, E. A. Phys. Fluids (2018)] focusing on the motion of a pair of point vortices impinging on a fixed point vortex. Here, in contrast, the pair of opposite-signed finite-size vortices, or vortex dipole for simplicity, can deform. This deformation has an impact on the dynamics. We show that, as expected, finite size vortices behave like point vortices if they are distant enough from each other. This allows one to recover the rich and diverse set of possible trajectories for the dipole. This includes the regimes of intricate bounded motion when the finite-size vortices remain stable near the fixed vortex for a long time. On the other hand, we show that large finite-size vortices can deform significantly and deviate from the trajectories of equivalent point vortices. When the shear that the vortices induce on each other is large enough, the finite size vortices may break into smaller structures or may even be completely strained out.

Keywords: finite size vortex, vortex interaction, vortex-topography interaction

\footnotetext{
a) Electronic mail: jnr1@st-andrews.ac.uk

b)Electronic mail: kvkoshel@poi.dvo.ru

c)Electronic mail: ryzhovea@gmail.com
} 


\section{INTRODUCTION}

The study of the evolution of opposite-signed vortex pairs has great practical importance as they transport momentum. Such structures have been the focus of a multitude of numerical and experimental studies including the recent works ${ }^{2-4}$. Here, we consider the motion of two opposite-signed, finite-size vortices of uniform vorticity, known as FAVOR ${ }^{5}$ (Finite Area VOrtex Region), interacting with a fixed point vortex. In practice, a fixed vortex can be used as part of the representation of an island or of isolated topography in geophysical fluid dynamics ${ }^{6-9}$. Furthermore, a vortex dipole is the simplest self-propelling coherent structure often observed in the ocean ${ }^{10,11}$. Vortex dipoles can transport water and its content for many kilometers. It is therefore both important and interesting to analyse the regular and chaotic dynamics they exhibit ${ }^{12-24}$. The evolution of a pair of uniform vorticity patches has been the topic of many previous works ${ }^{25-28}$. Such vortices are fully described by the contour bounding the vortex patches. The evolution of the flow can be studied following the dynamical evolution of the vortex bounding $\operatorname{contours}^{29}$.

The study presented in this paper is the natural extension of the study of the motion of two point vortices impinging on a fixed vortex reported in the accompanying paper ${ }^{1}$. The accompanying study evinces the dynamical richness of the configuration, even given the fact that the motion of the point vortices is integrable, hence regular. In particular, it is shown that the vortices can be entrapped in the vicinity of the fixed vortex, rotating around the fixed vortex for long times. We extend the study to finite size vortices. Finite size vortices have shapes, hence can be deformed by the shear and strain that the vortices exert on each other. This deformation has first an impact on the velocity field the vortices induce, hence on their motion. Moreover, large strain and shear rates can destroy a vortex, either partially or completely. This dynamical behaviour is prohibited in the equivalent point vortex model. It is therefore important to revisit the problem with finite size vortices.

We show that vortices whose size is much less than the typical distance separating the vortices behave, as expected, almost like point vortices and we recover similar trajectories as the ones obtained for point vortices. ${ }^{1}$ However, the trajectories of vortices of moderate size start to deflect from the trajectories of the equivalent point vortices. Moreover one of the finite size vortices can be sheared out by the intense shear that the fixed point vortex induces if it approaches close enough. Furthermore, a finite size vortex dipole can be itself 


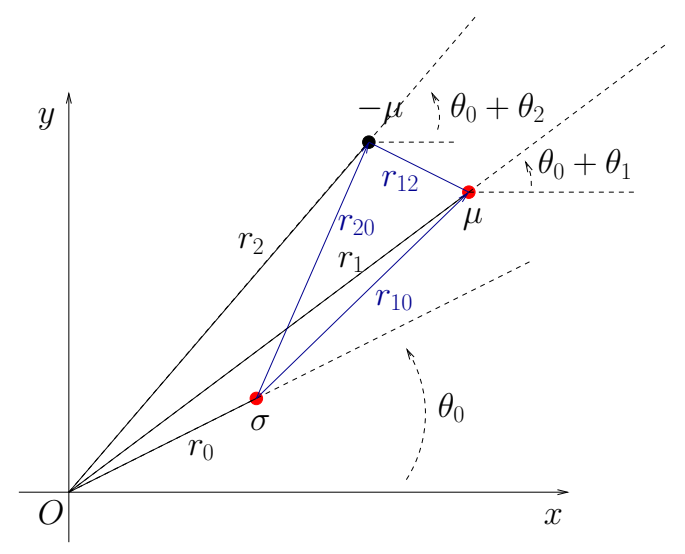

FIG. 1. General geometry of the flow configuration. The fixed vortex has index 0 . The vortices of the dipole have indices 1 and 2 .

unstable if its vortices are separated by a distanceless than a threshold. ${ }^{28}$ A finite size vortex dipole can thus be destroyed before it reaches the vicinity of the fixed vortex. The paper is organised as follows. Section 2 introduces the mathematical model and numerical set-up for the study. Results are presented in section 3, and some conclusions are proposed in section 4.

\section{MATHEMATICAL AND NUMERICAL SET-UP}

We consider a two-dimensional, inviscid, uniform density flow in the $(x, y)$-plane. The governing Euler's equations are recast in terms of vorticity and streamfunction,

$$
\begin{gathered}
\frac{\partial \omega}{\partial t}+u \frac{\partial \omega}{\partial x}+v \frac{\partial \omega}{\partial y}=0 \\
\frac{\partial^{2} \varphi}{\partial x^{2}}+\frac{\partial^{2} \varphi}{\partial y^{2}}=\omega \\
\boldsymbol{u}=\left(-\frac{\partial \varphi}{\partial y}, \frac{\partial \varphi}{\partial x}\right)
\end{gathered}
$$

where $t$ is time, $\omega$ is the vorticity and $\varphi$ the streamfunction and $\boldsymbol{u}=(u, v)$ is the velocity field. Equation (1) states that vorticity is materially conserved under our assumptions.

The general geometry of the problem is described in fig. 1. The fixed point vortex has circulation $\Gamma_{0}=2 \pi \sigma$ and is located at $\boldsymbol{x}_{0}=\left(x_{0}, y_{0}\right)=r_{0}\left(\cos \theta_{0}, \sin \theta_{0}\right)$. It induces a time-independent velocity field 


$$
\boldsymbol{u}_{0}(\boldsymbol{x})=\sigma \frac{\left(y_{0}-y, x-x_{0}\right)}{\left|\boldsymbol{x}-\boldsymbol{x}_{0}\right|^{2}}
$$

This velocity field can be readily obtained by inverting equation (2) for $\omega=\Gamma_{0} \delta\left(\left|\boldsymbol{x}-\boldsymbol{x}_{0}\right|\right)$, where $\delta$ is the Dirac distribution. The two finite size vortices have the same surface area $A_{v}$ and equal and opposite uniform vorticity $\pm \omega_{v}$, where $\omega_{v}>0$ without loss of generality. It is important to note that considering finite size vortices introduces a new length scale of the problem, associated with the vortex size compared to the point vortex problem. This length scale can be defined as $R=\sqrt{A_{v} / \pi}$, the vortex mean radius. The centroid of the finite size vortex $i=1,2$ is located at $\boldsymbol{x}_{i}=\left(x_{i}, y_{i}\right)=r_{i}\left(\cos \left(\theta_{0}+\theta_{i}\right), \sin \left(\theta_{0}+\theta_{i}\right)\right)$ where $\theta_{i}$ is the polar angle of vortex $i$ relative to the location of the fixed vortex. In this paper we indicate the locations of the vortices in the Cartesian or polar coordinates, whichever is most convenient. We denote the circulation of the finite size vortices $\pm \Gamma_{v}= \pm A_{v} \omega_{v}= \pm 2 \pi \mu$. Each finite size vortex induces a streamfunction

$$
\varphi_{v}(\boldsymbol{x})= \pm \mu \iint_{S_{v}} \ln \left|\boldsymbol{x}-\boldsymbol{x}^{\prime}\right| \mathrm{d} x^{\prime} \mathrm{d} y^{\prime}
$$

where $S_{v}$ is the surface of the vortex. The associated velocity field is

$$
\begin{aligned}
\boldsymbol{u}_{v}(\boldsymbol{x}) & =\left(-\frac{\partial \varphi_{v}}{\partial y}, \frac{\partial \varphi_{v}}{\partial x}\right) \\
& = \pm \mu \oint_{C_{v}} \ln \left|\boldsymbol{x}-\boldsymbol{x}^{\prime}\right| \mathrm{d} \boldsymbol{x}^{\prime}
\end{aligned}
$$

where the surface integral over the surface $S_{v}$ of the uniform vorticity vortex patch has been converted into a contour integral over the contour $C_{v}$ bounding the vortex, using Green's theorem. In practice, the contours bounding the vortices are discretized by nodes and the integration is done explicitly on local cubic splines between the nodes. ${ }^{30}$

For convenience we introduce the shorthand $r_{i j}=\left|\boldsymbol{x}_{i}-\boldsymbol{x}_{j}\right|, i$ and $j$ being the vortex of indices 0,1 or 2 . If $r_{10}=r_{20}$, and $\theta_{1}=-\theta_{2}$, the configuration is referred to as a symmetric case. The accompanying work ${ }^{1}$ distinguishes two general cases of the point-vortex equivalent system: (i) the symmetric case corresponds to the dipole's vortices initiated at the same distances from the fixed one - in this case only unbounded evolution of the point vortex dipole is possible (i.e. there are no bounded motion regimes); (ii) the asymmetric case 

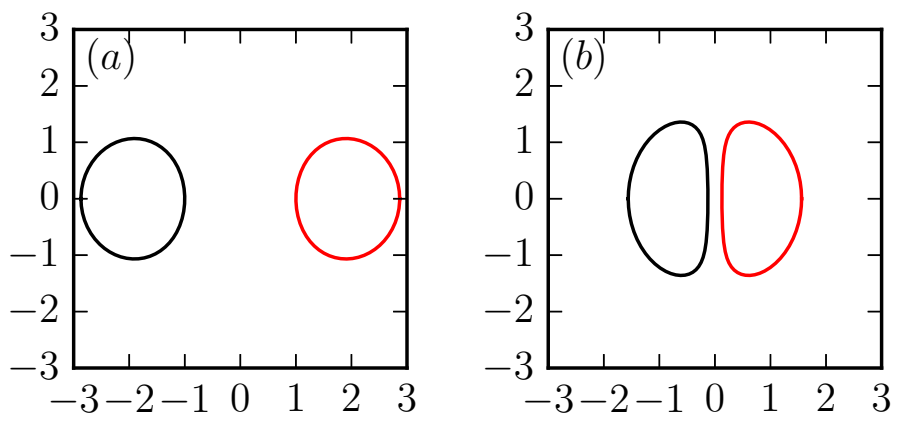

FIG. 2. Steadily translating uniform vorticity finite-core vortex dipoles with $A_{v}=\pi, \omega=2 \pi$ for $r_{12} / R=3.85(a)$, and $1.56(b)$.

corresponds to any initial vortex arrangement excluding the symmetric one - in this case both unbounded and bounded regimes of the point vortex dipole evolution are feasible.

We define the circulation ratio $\alpha=|\sigma / \mu|$. Finally, the time scale of the problem is given by $T=4 \pi / \omega_{v}$, corresponding physically to the turnover period of the circular vortex of uniform vorticity $\omega_{v}$.

When the finite size dipole is initially far away from the fixed vortex, it is convenient to use a pair of vortices in mutual equilibrium. Here we mean a pair of vortices steadily translating (without deforming) in the absence of external flow (i.e. in absence of fixed vortex). Using such an equilibrium avoids the artificial pulsation that vortices of any other shape would exhibit. Figure 2 shows examples of the shape of steadily translating uniformvorticity finite size vortex dipoles obtained for the purpose of the present study. These solutions are identical to those previously discussed in the literature. ${ }^{28,31}$

The nonlinear simulations are performed using the Contour Surgery numerical algorithm, with the standard set-ups ${ }^{30}$. Due to the presence of the singular fixed vortex and the associated high velocities in its vicinity, the time step $\Delta t$ used for the fourth order RungeKutta time integration is adaptive and is set to $\Delta t=\Delta t_{0} \min \left(1,\left|\boldsymbol{u}_{\max }(0) / \boldsymbol{u}_{\max }(t)\right|\right)$ where $\Delta t_{0}=\pi /\left(10 \omega_{v}\right)$ is the vorticity-controlled time step and $\left|\boldsymbol{u}_{\max }(t)\right|$ is the maximum velocity measured along the contours bounding the finite size vortices at time $t$.

In this work, we also analyse the evolution of the shape of the finite size vortices by determining their second order geometric moments defined in the symmetric $2 \times 2$-matrix 


$$
\mathcal{A}=\left(\begin{array}{cc}
I_{x x} & I_{x y} \\
I_{x y} & I_{y y}
\end{array}\right),
$$

where

$$
\begin{gathered}
I_{x x}=\frac{4}{A_{v}} \iint_{S_{v}}\left(x-x_{v}\right)^{2} \mathrm{~d} x \mathrm{~d} y, \\
I_{x y}=\frac{4}{A_{v}} \iint_{S_{v}}\left(x-x_{v}\right)\left(y-y_{v}\right) \mathrm{d} x \mathrm{~d} y, \\
I_{y y}=\frac{4}{A_{v}} \iint_{S_{v}}\left(y-y_{v}\right)^{2} \mathrm{~d} x \mathrm{~d} y,
\end{gathered}
$$

and $\left(x_{v}, y_{v}\right)=\boldsymbol{x}_{v}$ is the centroid of the vortex

$$
\boldsymbol{x}_{v}=\frac{1}{A_{v}} \iint_{S_{v}} \boldsymbol{x}^{\prime} \mathrm{d} x \mathrm{~d} y .
$$

These moments define the best-fit ellipse whose semi-axis lengths $a$ and $b$ are the square roots of the eigenvalues of the matrix $\mathcal{A}$ and whose orientation is given by the eigenvectors of $\mathcal{A}$. But convention, $a \geq b$ so that $a$ is the length of the major semi-axis while $b$ is the length of the minor semi-axis. In practice, the surface integrals in the formulae above are also converted to contour integrals over the vortex bounding contours.

\section{RESULTS}

\section{A. Influence of using equilibrium dipoles}

We start by illustrating the difference between the evolution of a pair of opposite-signed finite area vortices in mutual equilibrium and the evolution of initially circular vortex patches interacting with a fixed vortex. In all our numerical experiments, the area of the finite size vortices is set to $A_{v}=\pi$ so that their mean radius is $R=1$ unless stated otherwise. Their vorticity is $\omega_{v}= \pm 2 \pi$. The fixed vortex rotates in the clockwise direction and has circulation $\Gamma_{0}=-\pi^{2} / 2$ such that $\alpha=0.25$. The vortices are initially located at $\left(x_{0}, y_{0}\right)=(0,-5)$, $\left(x_{1}, y_{1}\right)=(-1.65,0)$ and $\left(x_{2}, y_{2}\right)=(1.65,0)$. Two simulations are performed with $(i)$ a dipole in mutual equilibrium for $r_{12} / R=3.3$ and $(i i)$ a dipole initially consisting of two circular vortices for the same relative distance. When the 'equilibrium' vortex pair is far away from the fixed vortex, the vortices evolve but remain in near equilibrium, contrarily to 

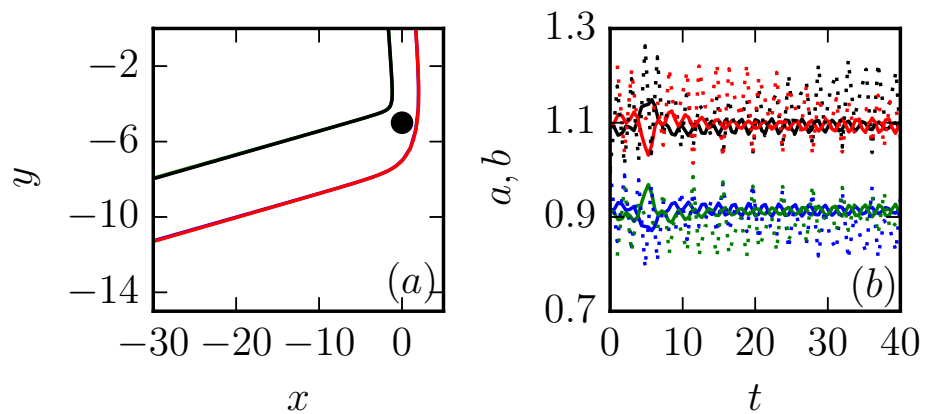

FIG. 3. Symmetric interaction: comparison between equilibrium vortices and circular vortices for $\alpha=0.25, r_{12} / R=3.3$ corresponding to $\left(x_{i}, y_{i}\right)=( \pm 1.65,0), i=1,2$ and $r_{0}=5 \theta_{0}=-\pi / 2$ corresponding to $\left(x_{0}, y_{0}\right)=(0,-5)$ at $t=0$. (a) trajectories of the vortex centres. $(b)$ evolution of the best-fit semi-axis lengths $a, b(a \geq b)$, for each vortex of the dipole vs $t$ for the near equilibrium vortices (solid lines) and initially circular vortex patches (dashes lines). The major semi-axis lengths $a$ of the two finite size vortices are in red and black while the minor semi-axis lengths of the two finite size vortices $b$ are in blue and green. The location of the fixed vortex is indicated by the black disk on the left panel.

the initially circular vortex patches. Results are shown in fig. 3 . The left panel of fig. 3 shows the trajectories of the vortex centroids. The trajectories for both cases are superimposed and almost identical. The evolution of the lengths $a, b$ of the semi-axes of the best-fit ellipses, shown on the right panel of fig. 3 is however significantly different. Results indeed showthat the oscillations are very small for the near equilibrium configuration. This is expected as the vortices deform almost quasi-statically. The peak deformation at $t \simeq 5$ corresponds to the time when the vortex dipole is nearest to the fixed vortex. In fact the vortices of the dipole pass on either side of the fixed vortex and this induces a strong perturbation on the dipole in near equilibrium. On the other hand, the initially circular patches are far from mutual equilibrium. As a result, they pulsate with high amplitude for all times. These oscillations can be seen as a direct consequence of the arbitrary choice of the (simple) shape for the vortices for which there is no physical justification. We therefore use equilibrium dipoles whenever it is relevant to do so and to avoid strong (artificial) pulsations for the vortices. 


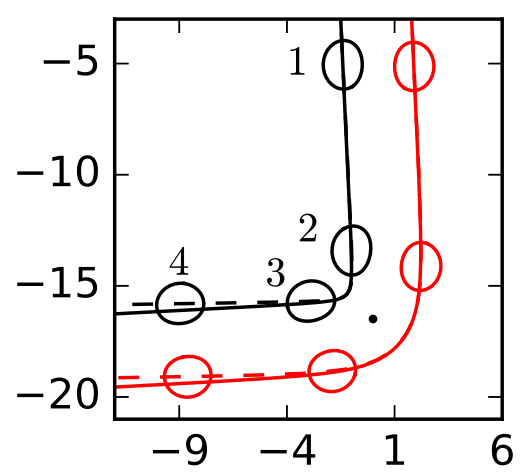

FIG. 4. Symmetric interactions: vortex bounding contours for finite size vortices with $r_{12} / r_{10}=$ $0.199, r_{12} / R=3.3(R=1)$ and $\alpha=0.25$ at (1) $t=5.43$ (2) 14.63 (3) $t=20.3$ and (4) $t=27.06$. The dashed lines indicate the trajectories of the finite size vortex centroids and the solid lines show the trajectories of equivalent point vortices.

\section{B. Characteristics of symmetric interactions}

We next explore some of the characteristics of the symmetric configurations $\left(r_{10}=\right.$ $\left.r_{20}, \theta_{1}=-\theta_{2}\right)$. For the purpose of illustration, we set $r_{12} / r_{10}=0.199$ at $t=0$. The vortex dipole is the same as the one used in the previous section, and corresponds to the equilibrium for $r_{12} / R=3.3$. Recall that here $R=1$. We first investigate the effect of varying the strength of the fixed vortex.

We start by considering a relatively weak fixed vortex with $\alpha=0.25$. Results are shown in fig. 4. The dipole is deflected by the fixed vortex by an angle of nearly $90^{\circ}$, and the finite size vortices follow very closely the trajectories of the equivalent point vortices. A slight deviation becomes apparent after the vortices pass on either side of the fixed vortex. This corresponds to the time the finite size vortices deform the most. It is worth noticing, however, that the finite core vortices behave qualitatively in the same way as the point vortices.

Increasing the circulation of the fixed vortex results in an increased deflection of the dipole. It also increases the shear and strain that the fixed vortex induces on the finite size vortices, and consequently the deformation of the finite size vortices increases. For $\alpha=0.5$, keeping the other parameters the same, the point vortex calculation predicts that the finite size vortex in co-rotation with the fixed vortex first moves directly towards it. Then it stops 

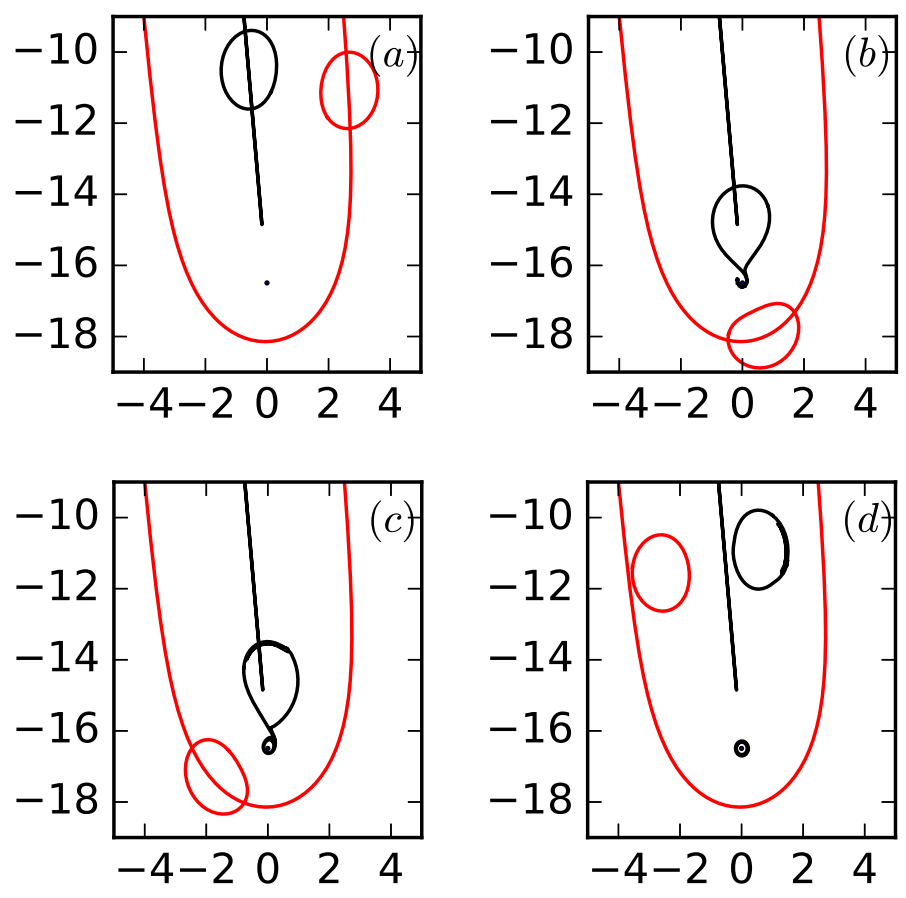

FIG. 5. Symmetric interactions: vortex bounding contours for finite size vortices with $r_{12} / r_{10}=$ $0.199, r_{12} / R=3.3(R=1)$ and $\alpha=0.5$ at $t=11.4(a), 17.3(b), 18.7(c), 23.5(d)$. The solid lines shows the trajectories of point vortices of the same circulation.

before reaching it. Finally it reverses its trajectory. Meanwhile the second vortex turns around the fixed vortex. ${ }^{1}$ The motion of the finite size vortices is illustrated in fig. 5 . The figure shows that the finite size vortices initially follow closely the trajectory of equivalent point vortices. When the finite size vortex heading towards the fixed vortex is close to it, it experiences intense shear and strain. As a consequence, a small filament of vorticity forms from the edge of the vortex and it wraps around the fixed vortex. Meanwhile, the second finite size vortex rotates around the fixed vortex. After the second finite size vortex completes a half turn around the fixed vortex, the interaction between the two finite size vortices is strong enough for the pair to leave the vicinity of the fixed vortex and resume a nearly rectilinear trajectory. A part of the filament originated from the vortex detaches from it and forms a small like-signed vortex ring which remains around the fixed vortex.

We next further increase the circulation of the fixed vortex to $\alpha=1$ while keeping $r_{12} / r_{10}=0.199$. We also use this set-up to illustrate the effect of the relative separation distance between the two finite size vortices of the dipole $r_{12} / R$. In all cases $R=1$ is used. 
Results are shown in fig. 6 for $r_{12} / R=5.94,5.43$ and 4.91, in fig. 9 for $r_{12} / R=3.85$ and in fig. 10 for $r_{12} / R=1.56$. In each case the dipole consists of a pair of vortices in mutual equilibrium for the given relative separation distance $r_{12} / R$. We also determine the trajectories of the equivalent point vortices (which is in fact invariant under the appropriate length scaling).

To understand the behaviour of the finite core dipole, we need first to describe qualitatively the relations between the deformation of the vortices and the velocity they induce. The far-field velocity induced by a finite size vortex can be expanded using a geometrical moment expansion ${ }^{5}$,

$$
\begin{gathered}
\psi_{v}(\boldsymbol{x})=\frac{\Gamma_{v}}{2 \pi} \ln \left|\boldsymbol{x}-\boldsymbol{x}_{i}\right|+ \\
\frac{\Gamma_{v}}{2 \pi} \frac{\left(\left(x-x_{i}\right)^{2}-\left(y-y_{i}\right)^{2}\right)\left(I_{x x}-I_{y y}\right)+4\left(y-y_{i}\right)\left(x-x_{i}\right) I_{x y}}{8\left|\boldsymbol{x}-\boldsymbol{x}^{\prime}\right|^{4}} \\
+\mathcal{O}\left(\left|\boldsymbol{x}-\boldsymbol{x}_{i}\right|^{-3}\right) .
\end{gathered}
$$

The leading order term corresponds to the contribution of an equivalent point vortex. It is also the external field induced by a uniform-vorticity, circular patch. The higher order terms take into account the shape of the vortex. These correction terms grow as the shape of the vortices departs from circles. Hence the discrepancy between the velocity induced by a finite size vortex and an equivalent point vortex increases as the deformation of the finite size vortex accumulates. The upshot is that there is a direct connection between the deformation of the vortices and the deviation of the finite size dipole trajectory from the one of an equivalent point vortex dipole.

Moreover, the shear induced at $\boldsymbol{x}$ by the fixed vortex scales as $\sigma /\left|\boldsymbol{x}-\boldsymbol{x}_{0}\right|^{2}$. For moderate relative size vortices (large $r_{12} / R$ ), the edges of the finite size vortices remain distant from the fixed vortex. The vortices remain compact patches of uniform vorticity. The amplitude of their relative deformation increases as $r_{12} / R$ decreases. Note that the increase in deformation has two origins. First, for small $r_{12} / R$ the equilibrium dipole is more deformed to allow the finite size vortices to withstand quasi-steadily the strain they induce on each other. On the other hand, decreasing $r_{12} / R$ with $r_{12} / r_{10}$ fixed implies that the finite size vortices can get closer to the fixed vortex, hence they can experience higher strain rates. As a consequence of the combination of these two effects, the deflection of the finite size dipole trajectory from 

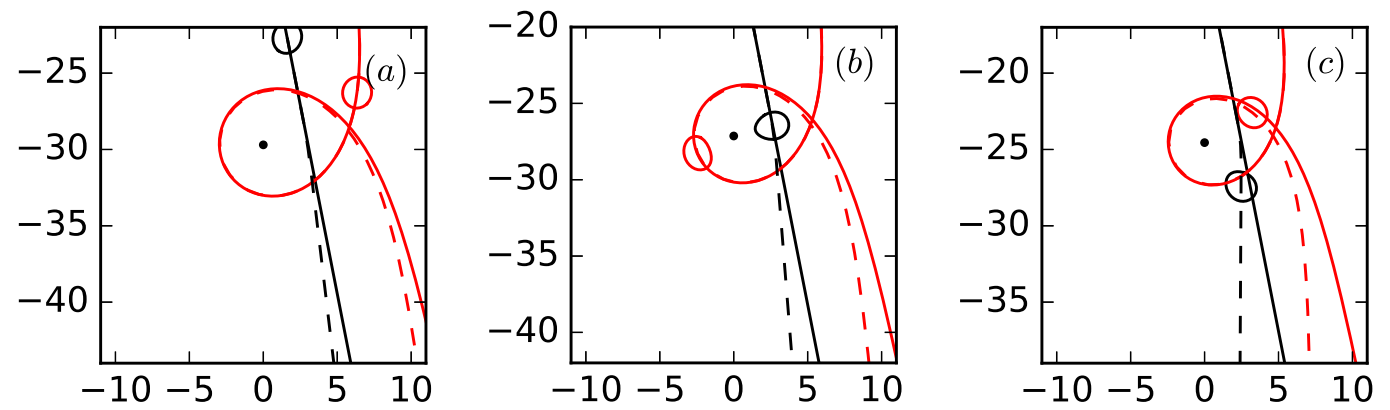

FIG. 6. Trajectories of the finite size vortex centroids (dashed lines) and the equivalent point vortices (solid lines) $r_{12} / r_{10}=0.199$ and $r_{12} / R=5.94(a), 5.43(b)$ and $4.91(c)$. The black dot indicates the location of the fixed vortex. The vortex bounding contours are shown at $t=43.8$ in panel $(a), t=46.6$ in panel $(b)$ and $t=43.2$ in panel $(c)$.

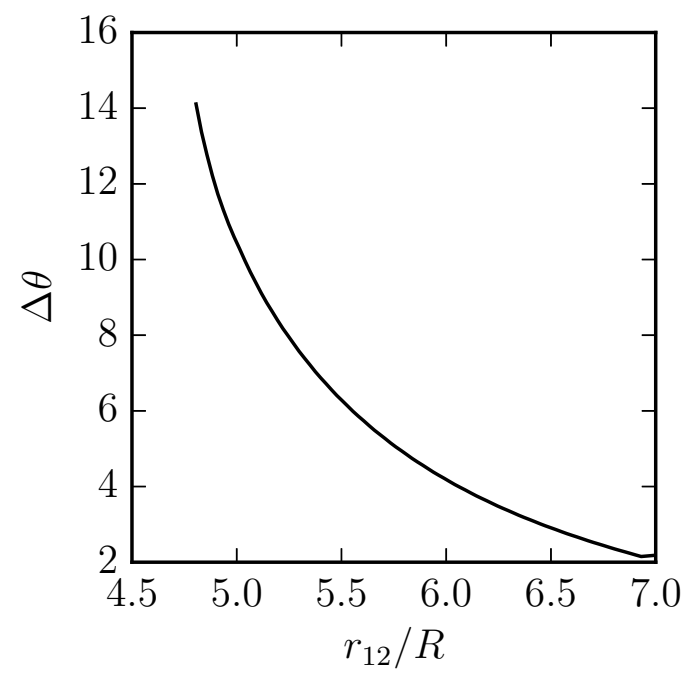

FIG. 7. Deviation angle difference between the point vortex case and the finite size case (measured in degrees) vs $r_{12} / R$ for $\alpha=1, r_{12} / r_{10}=0.199$.

the trajectory of an equivalent point-vortex dipole grows as $r_{12} / R$ decreases as seen in fig. 6 . Figure 7 shows, for a range of $r_{12} / R, \Delta \theta$ the difference between the angles of the trajectories of the point and finite size vortex dipoles, far away from the fixed vortex at a late time. Far from the influence of the fixed vortex, the vortex dipole resumes translating motion. A regression on the curve indicates that $\Delta \theta \propto r_{12}^{-4.85}$ in this case. Figure 8 shows the evolution of the best-fit ellipse semi-axis lengths $a$ and $b$ for both vortices and for the three cases $r_{12} / R=5.94,5.43$, and 4.91 . They all exhibit a strong peak corresponding to the time the 

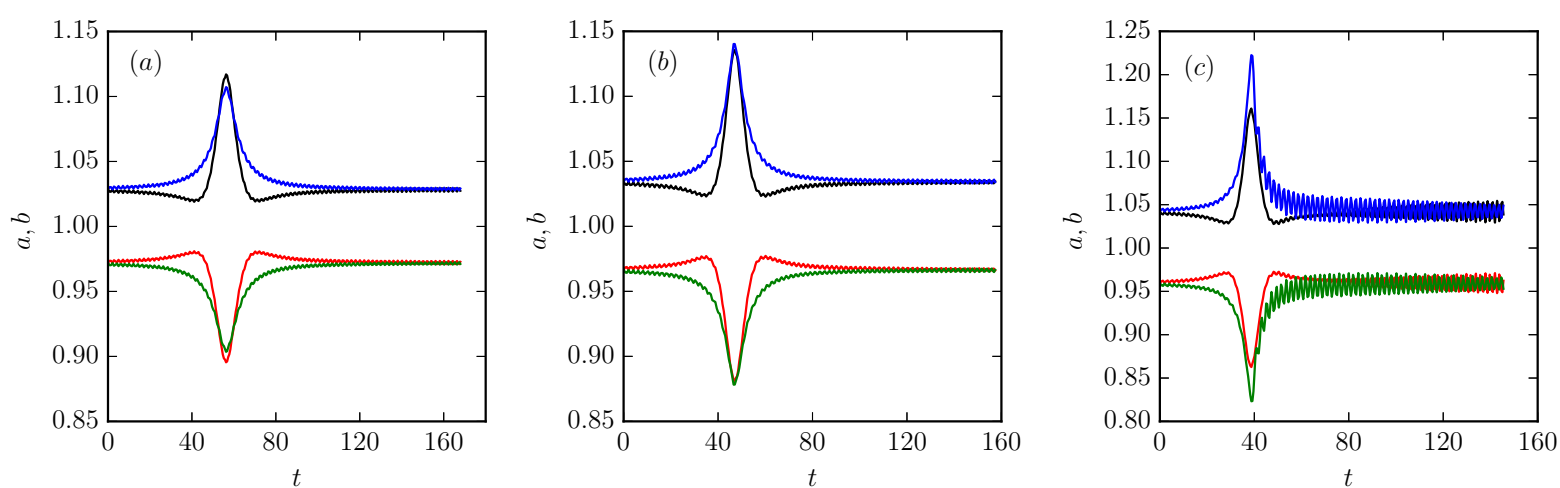

FIG. 8. Evolution of the best fit ellipse semi-axis lengths for $\alpha=1, r_{12} / r_{10}=0.199$ and $r_{12} / R=$ $5.94(a), 5.43(b)$ and $4.91(c)$.

dipole passes on either side of the fixed vortex. They also include higher frequency - low amplitude oscillations corresponding to the pulsation of the vortices. The vortices pulsate as they attempt to remain in a quasi-equilibrium state in the time-dependent external flow they are subject to. The amplitude of these pulsations remains significantly less than the ones observed for the initially circular vortex patches in a similar situation. The frequency of the oscillations $f=1 / T_{\text {osc }} \sim 0.5$ seems to only weakly vary with $r_{12} / R$ and it is likely to be associated with $\omega_{v}^{-1}$ which sets an internal time scale $T_{v}=4 \pi / \omega_{v}=2$ for the vortices.

For smaller $r_{12} / R$, the vortex moving closer to the fixed vortex sheds a filament when entering its vicinity. Appendix A provides an estimate of the shear rate sufficient for the generation of a filament by investigating the evolution of a single, initially circular, patch of vorticity near a fixed vortex. The estimate shows that a shear rate of the same order as vorticity of the vortex patch is enough for filamentation to occur. It should be noted however that the problem of the dipole moving towards the fixed vortex is non trivial as not only the distance between the dipole and the fixed vortex is time-dependent but the ability of a vortex to withstand shear depends on its shape.

As $r_{12} / R$ is further decreased a large part of the finite size vortex in co-rotation with the fixed vortex wraps around it, as seen in fig. 9. Eventually this finite size vortex splits and a secondary vortex resulting from the split pairs with the second, opposite-signed finite size vortex. The resulting asymmetric dipole travels away from the fixed vortex. Due to the loss of material, the vortices of the new dipole have different circulations. They do no resume a quasi-steady linear translation far from the fixed vortex but rather rotate about 

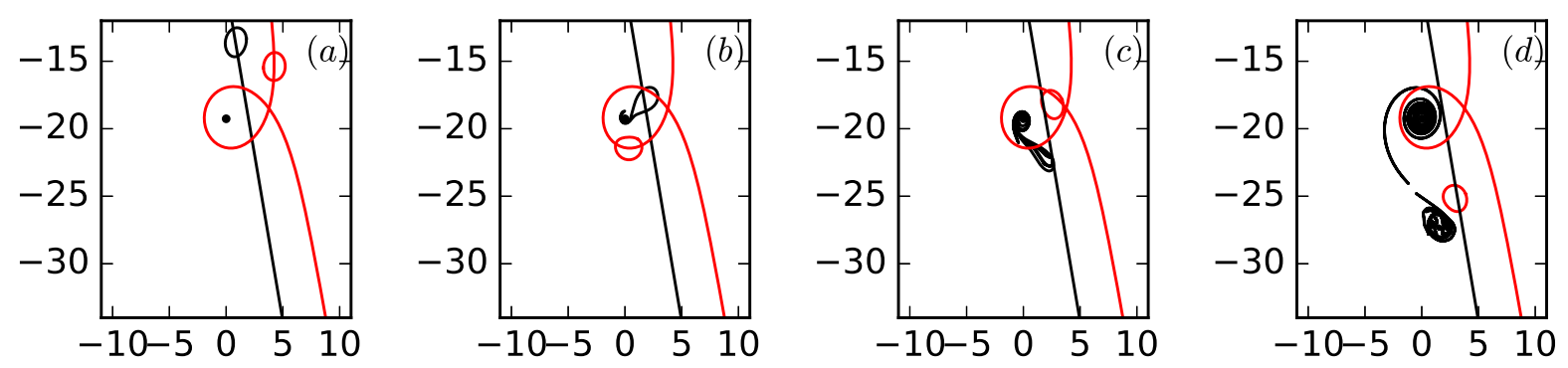

FIG. 9. Symmetric configuration. Evolution of the finite size dipole for $\alpha=1, r_{12} / r_{10}=0.199$, $r_{12} / R=3.85$ at $t=17.1(a), 22.5(b), 26.7(c)$ and $32.8(d)$. The solid lines indicate the trajectories of the equivalent point vortices.
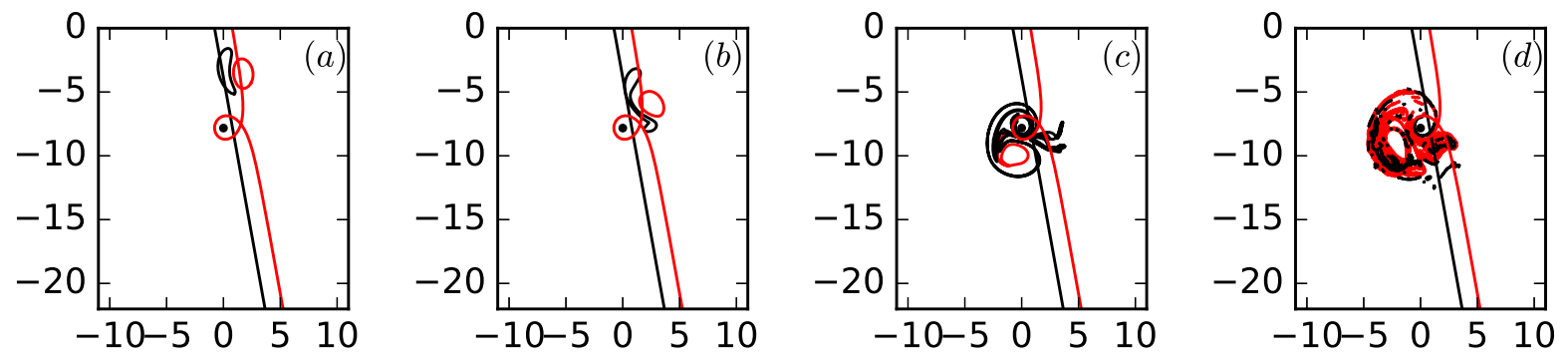

FIG. 10. Symmetric configuration. Evolution of the finite size dipole for $\alpha=1, r_{12} / r_{10}=0.199$, $r_{12} / R=1.56$ at $t=2.06(a), 3.44(b), 7.78(c)$ and $18.7(d)$. The solid lines indicate the trajectories of the equivalent point vortices.

their distant centre of vorticity.

Finally for small $r_{12} / R$ the dipole itself is unstable. It is perturbed by the velocity field induced by the fixed vortex and it destabilises. The vortex in co-rotation with the fixed vortex becomes closely surrounded by two other vortices (of different sign) and is sheared by the interaction. Parts of this vortex wrap around both the surrounding vortices into thin filaments. The second finite size vortex is also close to the fixed vortex at this stage. Due to the weakening of influence of the sheared vortex, the opposite-signed finite size vortex is also trapped by the fixed vortex, see fig. 10. It should be reminded that the non-deformable equivalent point vortices escape to infinity instead in this case. 


\section{Vortex entrapment for asymmetric configurations}

We next consider asymmetric configurations, $r_{10} \neq r_{20}$ at $t=0$. Only a few special cases where the vortices are entrapped in the vicinity of the fixed vortex are considered. We start with a configuration for which $\theta_{1}=\theta_{2}=\pi$ at $t=0$. This means that the two finite size vortices and the fixed vortex are initially aligned. It should be noted that the vortices in the equivalent point-vortex configuration align periodically during their evolution. ${ }^{1}$ For the sake of simplicity we set $r_{12} / R=3.85$ for all cases and we vary $r_{10}$. The equilibrium dipole itself is stable for this separation. Results for $\alpha=1 r_{10} / R=6.07,5.07,4.07$ are presented in fig. $11(a)-(c)$. The vortex closest to the fixed vortex is designated as vortex 1. The dipole starts to travel in the direction dictated by its polarity, with a positive angular velocity in the present case. Then vortex 1 gets closer to the opposite-signed fixed point vortex, and their mutual interaction becomes dominant for the motion of vortex 1 . This results in the finite size vortices reversing their direction of motion until vortex 1 and vortex 2 pair again. This happens when vortex 1 and 2 become close enough. This scenario repeats itself periodically. Results are qualitatively similar to those obtained for point vortices. The finite size vortices both change the direction of their motion near the location of their minimum distance possible from the fixed vortex. This explains the small loops observed in their trajectories (this regime corresponds to regime 3 in the accompanying paper ${ }^{1}$, see in particular their fig. 8). As the distance between vortex 1 and the fixed vortex is decreased, i.e. as $r_{10} / R$ is decreased, their interaction dominates the overall motion for longer periods of time, hence for larger parts of the full trajectory. For $r_{10} / R=3.07$, also shown in fig. 11 $(d)$, vortex 1 is close enough to the fixed vortex for their mutual interaction to force vortex 1 to rotate around it in the same direction at all times. Vortex 2 however still reverses its trajectory periodically (this regime corresponds to regime 2 in the accompanying paper ${ }^{1}$, see in particular their fig. 8). Its direction of motion depends on whether the like-signed fixed vortex or the opposite-signed finite size vortex dominates the velocity affecting vortex 2 . This in turn depends on the location of the opposite-signed finite size vortex, in particular on which side of the fixed vortex it lies comparing to the location of vortex 2 .

For $r_{10} / R=2.07$, presented in fig. 12 , the inner finite size vortex 1 , in counter-rotation with the fixed vortex, sheds a filament due to the shear induced by the fixed vortex. The amount of material lost by the vortex is small and the vortices continue their bounded 

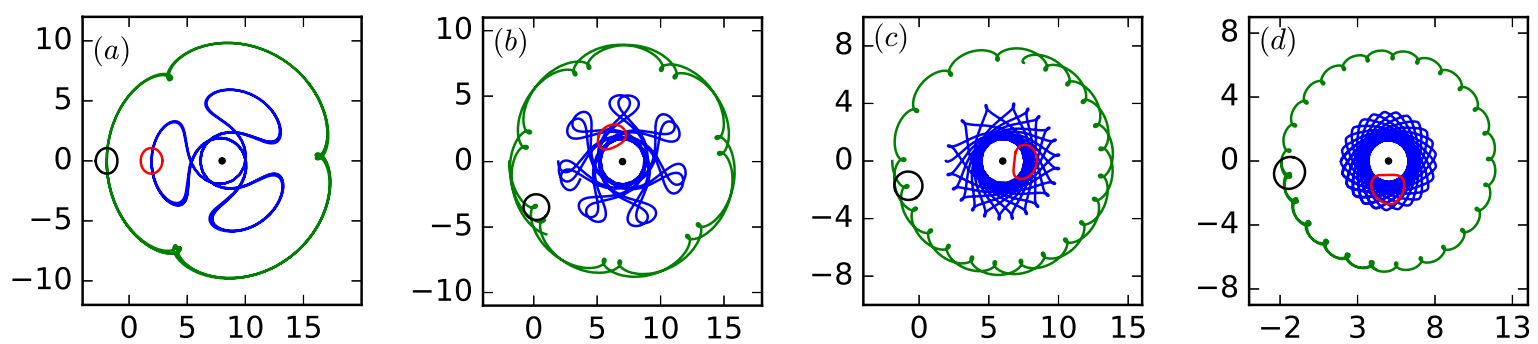

FIG. 11. Asymmetric configuration. Trajectories of the finite size dipole (solid blue and green lines) for $\alpha=1, \theta_{1}=\theta_{2}=\pi, r_{12} / R=3.85$ and $r_{10} / R=6.07(a), 5.07(b), 4.07(c)$ and $3.07(d)$. The finite size vortex bounding contours are shown at $t=0$ in panel $(a), 13.3$ in panel $(b), 9.34$ in panel $(c)$ and 6.59 in panel $(d)$.
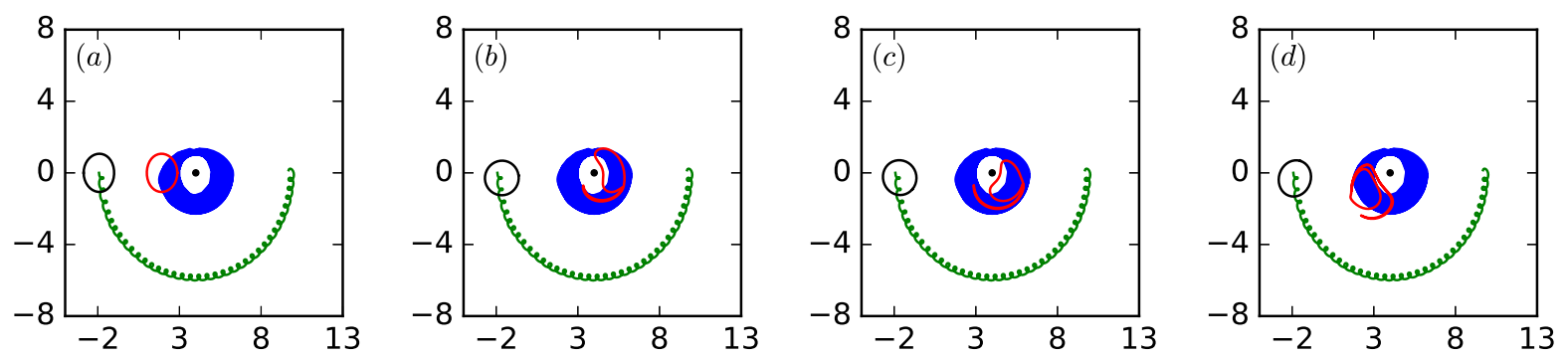

FIG. 12. Asymmetric configuration. Trajectories of the finite size dipole (solid blue and green lines) for $\alpha=1, \theta_{1}=\theta_{2}=\pi r_{12} / R=3.85$ and $r_{10} / R=2.07$ at $t=0(a), 3(b), 3.2(c)$ and $4.6(d)$.

motion.

For $r_{10} / R=1.07$, the edge of one of the finite size vortices is close to the fixed vortex and the vortex is simply wrapped around the fixed one. Since $\alpha=1$, the circulation of the vortex wrapping around the fixed vortex is equal and opposite to the circulation of the fixed vortex. The compound structure consisting of the fixed vortex and the opposite-signed finite vortex wrapped around it has an overall vanishing circulation which has very little influence on vortex 2. At late times however, some of the vorticity of the wrapping vortex drifts close to vortex 2 and they form a secondary dipolar structure. The upshot of the interaction is the partial destruction of one of the finite size vortices as seen in fig. 13.

We next investigate the influence of the relative size of the vortices. This is illustrated on cases with $\alpha=3, r_{12}=8.125$ and $r_{20}=50, \theta_{1}=\theta_{2}=0\left(r_{10} / r_{20}=0.835\right)$. Results are presented in fig. 14 for $R=1,2,3$, and 4 . In each case, a finite size dipole is initialised 

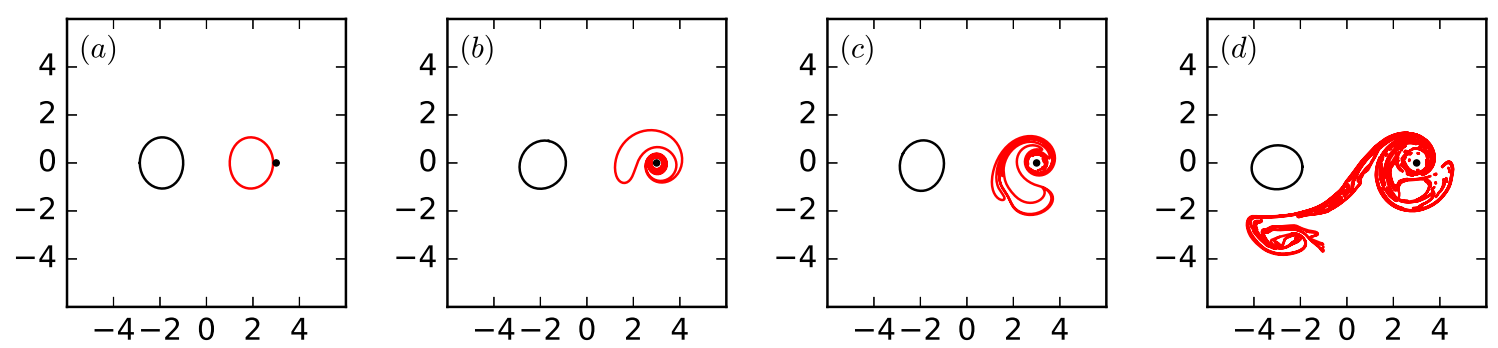

FIG. 13. Asymmetric configuration. Trajectories of the finite size dipole (solid blue and green lines) for $\alpha=1, \theta_{1}=\theta_{2}=\pi r_{12} / R=3.85$ and $r_{10} / R=1.07$ at $\alpha=1 t=0(a), 0.6(b), 2.2(c)$ and $9(d)$.
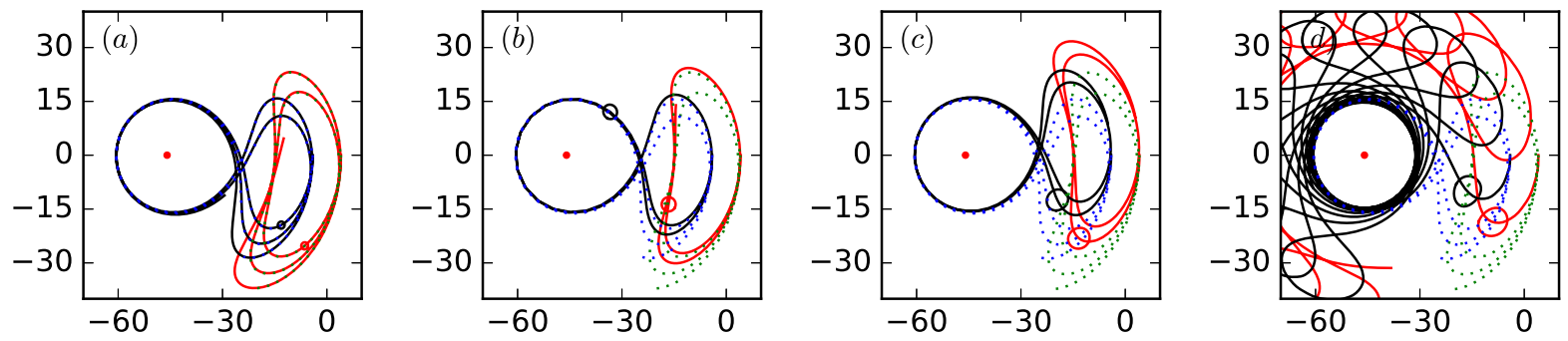

FIG. 14. Asymmetric configuration. Trajectories of the finite size dipole's vortex centroids (solid lines) for $\alpha=3, \theta_{1}=\theta_{2}=0 r_{12}=8.125$ and $r_{20}=50 ; R=1(a), R=2(b) R=3(c)$ and $R=4(d)$. The dashed lines indicate the trajectories of the equivalent point vortices.

in equilibrium. For small vortices $(R=1)$ the trajectories of the finite size vortices are almost identical to the ones of the equivalent point vortices. Similarly to the previous, the deflection of the trajectory of the finite size dipole from the trajectory of the point vortex dipole increases as $R$ increases for fixed $r_{12}$. This is, as before, the direct consequence of the increased deformation of the equilibrium finite size vortex dipole.

If both finite size vortices are located close to an intense fixed vortex, their angular velocity is dominated by the one induced by the fixed vortex, and both finite vortices rotate in the same direction for all times. An example of such motion is provided in fig. 15 (this regime corresponds to regime 1 in the accompanying paper ${ }^{1}$, see in particular their fig. 8) for $\alpha=3, r_{12} / R=3.85$ and $r_{12} / r_{10}=1, \theta_{1}=\theta_{2}=0$, hence $r_{20}=r_{12}+r_{10}=7.7$. In this situation the trajectories of the finite size vortices are again qualitatively similar to the ones obtained for point vortices in the same regime. The example however shows again that the 

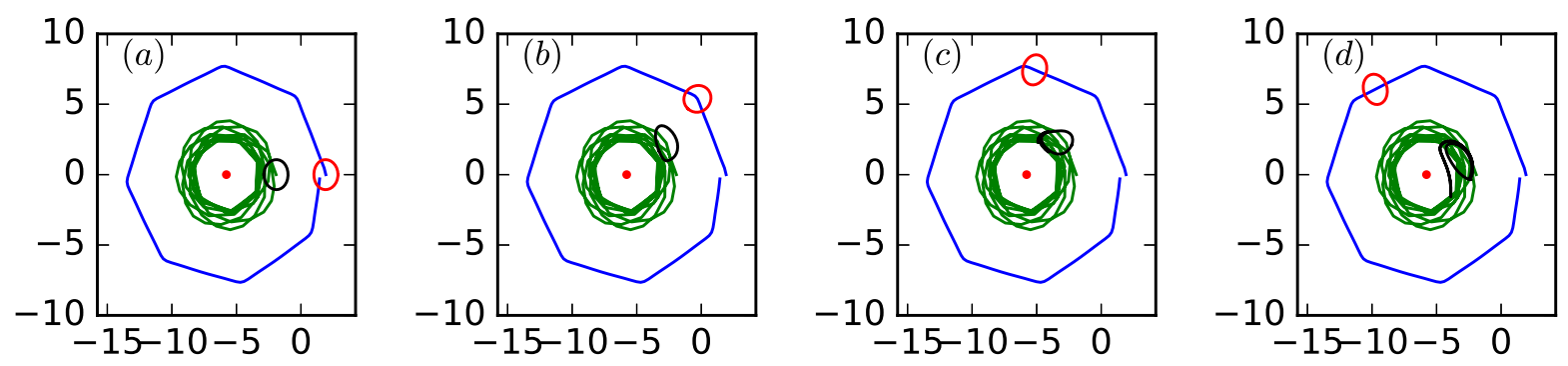

FIG. 15. Asymmetric configuration. Trajectories of the finite size dipole's vortex centroids (solid blue and green lines) for $\alpha=3, r_{12} / R=3.85$ and $r_{12} / r_{10}=1$ and vortex bonding contours (solid black and red lines) at $t=0(a), 7.3(b), 13.8(c)$ and $20.3(d)$.
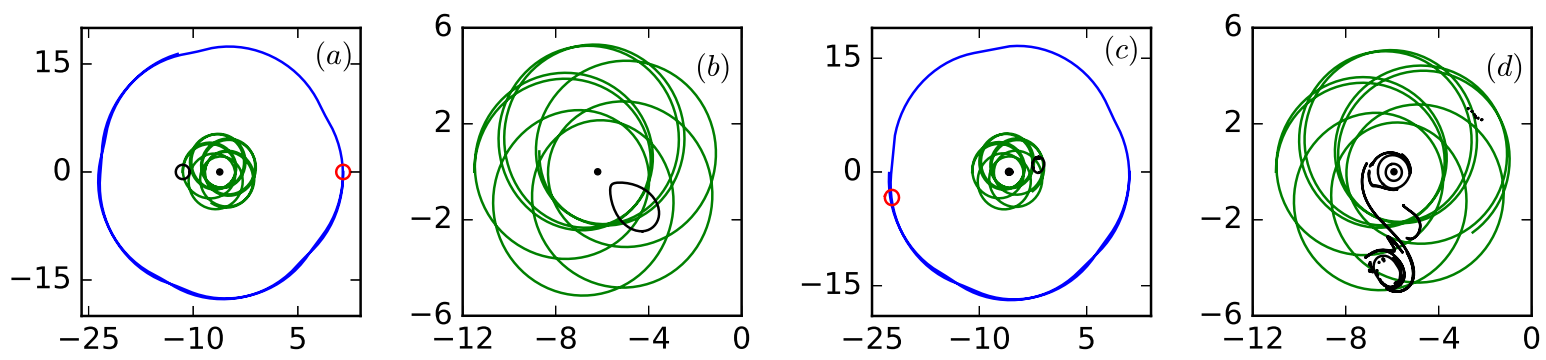

FIG. 16. Asymmetric case: Trajectories of the finite size vortex centroids for $\alpha=0.5, r_{10} / r_{20}=0.3$ with $\theta_{1}=\pi, \theta_{2}=0$ at $t=0$ and $r_{12} / R=23$ in panels $(a)$ and $(b)$ and $r_{12} / R=22$ with for panels $(c)$ and $(d)$. The vortex bounding contours are indicated in the figure at $t=0$ in panel $(a), 46.7$ (b) and $t=193(c)$ and $360(d)$.

finite size vortex dipole can be subject to strong deformation and can even shed filamentary vorticity as the waves travelling along the edge of the vortices can grow nonlinearly.

In the last examples, both finite size vortices rotate around the fixed vortex in opposite direction and never reverse their direction. Vortex 1, the closest to the fixed vortex, is in corotation with it while the outer finite size vortex (vortex 2) travels in the opposite direction. In these cases, the distance between the inner vortex (vortex 1) and the fixed vortex is smaller than the distance between the two finite size vortices. The interaction between the inner vortex and the fixed vortex is stronger than the interaction between the two finite size vortices, therefore using the finite size dipole in mutual equilibrium is irrelevant. We therefore use circular patches of uniform vorticity as initial conditions for the finite size dipole. Results are shown in fig. 16 for $\alpha=0.5$ and $r_{10} / r_{20}=0.3, \theta_{1}=\pi$ and $\theta_{2}=0$ and 
two values of $r_{12} / R$. This regime corresponds to regime 4 in the accompanying paper ${ }^{1}$, see in particular their fig. 8. For $r_{12} / R=23$, both finite size vortices remain distant enough from the other vortices to remain compact patches of vorticity at all times. Vortex 1 can however exhibit a near sharp inner edge as it reaches its minimum separation distance from the fixed vortex, see in particular fig. 16(b). For smaller $r_{12} / R=22$, shown in fig. $16(c)-(d)$, the inner finite size vortex is close enough to the fixed vortex and it sheds filaments which wrap around the fixed vortex.

There are other regimes of motion ${ }^{1}$ but they are typically associated with well-separated vortices hence no significant differences between the point vortex configurations and the finite size vortices are expected unless the inner vortex comes close to the fixed vortex and is strained out.

\section{CONCLUSION}

We have investigated the motion of a pair of counter-rotating finite-size vortices impinging on a fixed vortex. For the sake of simplicity the fixed vortex was taken as a singular point vortex while the pair of counter-rotating vortices free to move had finite size. Finite size vortices have the ability to deform when subject to external shear and strain. The deformation of a vortex depends on the ratio of its vorticity to the external deformation rate. For a given circulation, large vortex patches deform more profoundly than smaller vortices. This deformation of the vortex implies the modulation of the external velocity field they induce. Indeed only a non-deformable circular patch induces an external velocity field equal to the one of a point vortex of the same circulation. As interacting vortices in a non-equilibrium set-up deform, finite size vortices exhibit dynamics which differ from the ones of the equivalent point vortices. The discrepancy between the trajectories increases with the deformation of the vortices hence with the ratio of the sizes of the vortices to the distance separating them.

It should be noted however that as expected all the trajectories observed in the accompanying investigation of the equivalent point vortex system ${ }^{1}$ can be recovered with finite size vortices. Therefore, it is definite that these complex motion regimes are not artefacts of the singular vortex approach. For instance, the finite-size vortex model effectively captures the periodic motion regimes when the finite-size vortices similar to the point ones oscillate near 
the fixed vortex in a non-trivial way remaining stable for a long time.

On the other hand, vortices can not only deform in a quasi-static way but they can also deform irreversibly by shedding filamentary vorticity of even break into secondary structures. This happens whenever the external deformation rate applied to the vortices exceeds a threshold depending on the vortex characteristics. In this case one or both vortex can be partially destroyed affecting significantly their motion.

A natural extension of this work concerning oceanographic applications is to consider the motion of a vortex pair impinging on a finite size submerged obstacle instead of the one considered here modelled by a point vortex. In this case, the evolution of the vortex pair will be the same in the far field, but noticeably differing near and directly over the obstacle ${ }^{32}$. However, a number of periodic and quasi-periodic solutions reported here may still remain.

\section{ACKNOWLEDGMENTS}

The reported study was partially supported the POI FEB RAS Program "Mathematical simulation and analysis of dynamical processes in the ocean" $(117030110034-7)$ and by the Russian Foundation for Basic Research, project no. 17-05-00035. EAR was partially supported by the NERC grant $N E / R 011567 / 1$. The work of KVK in comparing trajectories of the point vortices and the finite-size ones was supported by the Russian Scientific Foundation, project no. $16-17-10025$.

\section{Appendix A: Filament generation}

We consider a simple configuration to evaluate the shear necessary for a finite area vortex to shed a filament. We consider a circular patch of uniform vorticity of radius $R=1$ and vorticity $\omega_{v}=2 \pi$ (hence circulation $\Gamma_{v}=2 \pi^{2}$ ) initially located at $(0,0)$ and a fixed point vortex located at $(0,2 R)=(0,2)$ and of circulation $\Gamma_{0}=\alpha \Gamma_{v}=2 \pi^{2} \alpha$. The edge of the vortex patch is at a distance $d=R=1$ of the fixed vortex such that the shear induced by the point vortex on the finite size vortex edge scales as $s=\Gamma_{0} / d^{2}$ so that $s / w_{v}=\alpha \pi$.

For $s / w_{v} \gtrsim 0.32 \pi$, a filament is shed by the vortex. The filament generation is linked to the nonlinear growth of a vortex (Rossby) wave on the vortex boundary. ${ }^{33,34}$ For $s / w_{v} \gtrsim$ $0.36 \pi$ the filament generated is large enough to extend to the close vicinity of the fixed 

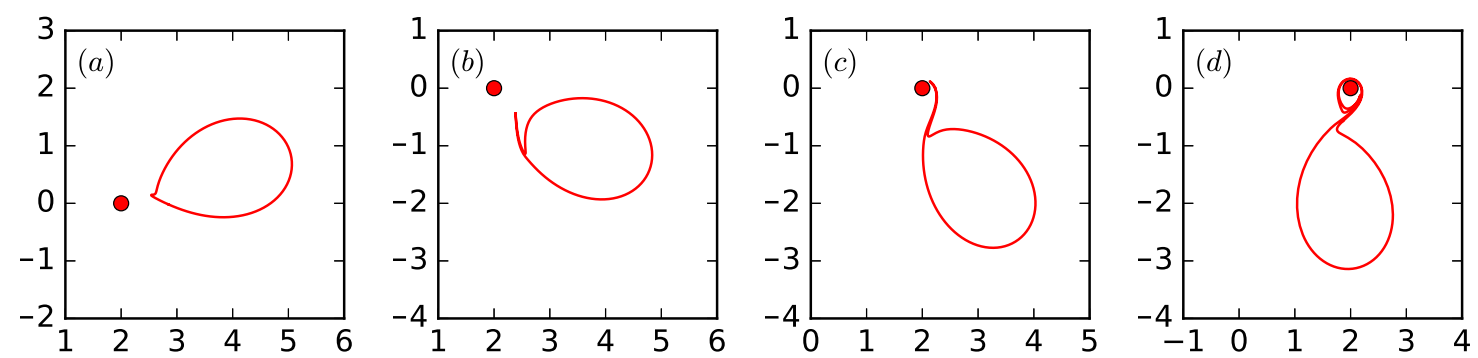

FIG. 17. Single vortex patch (initially circular) with $R=1$ with $\omega_{v}=2 \pi$ initially located at $(0,0)$ and a fixed point vortex at $(0,2)$ with $\alpha=0.3$ at $t=14$ in panel $(a), \alpha=0.32$ at $t=9.86$ in panel (b), $\alpha=0.34$ at $t=7.44$ in panel $(c)$ and $\alpha=0.36$ at $t=5$ in panel $(d)$.

vortex and to start wrapping around it.

\section{REFERENCES}

${ }^{1}$ K. V. Koshel, J. N. Reinaud, G. Riccardi, and E. A. Ryzhov, "Dynamics of a vortex pair interacting with a fixed point vortex revisited. Part I: Point vortices," Phys. Fluids 30 (2018).

${ }^{2}$ J. Tunkeaw and W. Rojanaratanangkule, "Effect of external turbulence on the shortwavelength instability of a counter-rotating vortex pair," Phys. Fluids 30 (2018).

${ }^{3}$ V. Sadri and K. P. S., "Pinch-off of axisymmetric vortex pairs in the limit of vanishing vortex line curvature," Phys. Fluids 28, 071701 (2016).

${ }^{4}$ E. A. Ryzhov and M. A. Sokolovskiy, "Interaction of a two-layer vortex pair with a submerged cylindrical obstacle in a two layer rotating fluid," Phys. Fluids 28, 056602 (2016). ${ }^{5}$ M. V. Melander, N. J. Zabusky, and A. S. Styczek, "A moment model for vortex interactions of the two-dimensional Euler equations. Part 1. Computational validation of a Hamiltonian elliptical representation," J. Fluid Mech. 167, 95-115 (1986).

${ }^{6}$ E. R. Johnson and N. R. McDonald, "The point island approximation in vortex dynamics," Geophys. Astrophys. Fluid Dyn. 99, 49-60 (2005).

${ }^{7}$ G. G. Sutyrin, X. Perrot, and X. Carton, "Integrable motion of a vortex dipole in an axisymmetric flow," Phys. Lett. A 372, 5452-5457 (2008).

${ }^{8}$ E. A. Ryzhov and K. V. Koshel, "Dynamics of a vortex pair interacting with a fixed point vortex," EPL 102, 44004 (2013). 
${ }^{9}$ E. A. Ryzhov, "Irregular mixing due to a vortex pair interacting with a fixed vortex," Phys. Lett. A 378, 3301-3307 (2014).

${ }^{10}$ P. L'Hégaret, X. Carton, I. Ambar, C. Menesguen, B. Hua, L. Chérubin, A. Aguiar, B. Le Cann, N. Daniault, and N. Serra, "Evidence of Mediterranean Water dipole collision in the Gulf of Cadiz," J. Geophys. Res. 119, 5337-5359 (2014).

${ }^{11}$ C. W. Hughes and P. I. Miller, "Rapid water transport by longlasting modon eddy pairs in the Southern midlatitude oceans," Geophys. Res. Lett. 44, 12375-12384 (2017).

${ }^{12}$ R. W. Griffiths and E. J. Hopfinger, "Experiments with baroclinic vortex pairs in a rotating fluid," J. Fluid Mech. 173, 501-518 (1986).

${ }^{13}$ V. V. Meleshko, M. Y. Konstantinov, A. A. Gurzhi, and T. P. Konovaljuk, "Advection of a vortex pair atmosphere in a velocity-field of point vortices," Phys. Fluids A-Fluid Dynamics 4, 2779-2797 (1992).

${ }^{14}$ Y. D. Afanasyev, S. I. Voropayev, and A. A. Kudin, "Vortex dipole inside a homogeneous intrusion in a density stratified fluid," Izvestiya, Atmospheric and Oceanic Physics 28, 253-259 (1992).

${ }^{15}$ D. Walsh and L. J. Pratt, "The interaction of a pair of point potential vortices in uniform shear," Dyn. Atmos. Oceans 22, 135-160 (1995).

${ }^{16}$ S. Y. Legg, H. Jones, and M. Visbeck, "A heton perspective of baroclinic eddy transfer in localized open ocean convection," J. Phys. Oceanogr. 26, 2251-2266 (1996).

${ }^{17}$ E. Y. Bannikova, V. M. Kontorovich, and G. M. Reznik, "Dynamics of a vortex pair in radial flow," J. Exp. Theor. Phys. 105, 542-548 (2007).

${ }^{18}$ G. Reznik and Z. Kizner, "Two-layer quasi-geostrophic singular vortices embedded in a regular flow. part 1. invariants of motion and stability of vortex pairs," J. Fluid Mech. 584, 185-202 (2007).

${ }^{19}$ P. Newton and H. Shokraneh, "Interacting dipole pairs on a rotating sphere," Proc. R. Soc. A 464, 1525-1541 (2008).

${ }^{20}$ K. Higgins, M. R'utten, A. Ooi, and M. S. Chong, "The interaction of counter-rotating strained vortex pairs with a third vortex," Fluid Dyn. Res. 41, 035502 (2009).

${ }^{21}$ R. Trieling, R. Santbergen, G. J. van Heijst, and Z. Kizner, "Barotropic elliptical dipoles in a rotating fluid," Theor. Comput. Fluid Dyn. 24, 111-115 (2010).

${ }^{22}$ J.-R. Angilella, "Asymptotic properties of wall-induced chaotic mixing in point vortex pairs," Phys. Fluids 23, 113602 (2011). 
${ }^{23} \mathrm{~K}$. V. Koshel and E. A. Ryzhov, "Parametric resonance with a point-vortex pair in a nonstationary deformation flow," Phys. Lett. A 376, 744-747 (2012).

${ }^{24}$ S. Llewellyn Smith and R. J. Nagem, "Vortex pairs and dipoles," Regul. Chaotic Dyn. 18, 194-201 (2013).

${ }^{25}$ M. V. Melander, N. J. Zabusky, and J. C. McWilliams, "Symmetric vortex merger in two dimensions: causes and conditions," J. Fluid Mech. 195, 303-340 (1988).

${ }^{26} \mathrm{D}$. W. Waugh, "The efficiency of symmetric vortex merger," Phys. Fluids A 4, 1745-1758 (1992).

${ }^{27}$ D. G. Dritschel and D. W. Waugh, "Qualification of the inelastic interaction of unequal vortices in two-dimensional vortex dynamics," Phys. Fluids A 4, 1737-1744 (1992).

${ }^{28}$ D. G. Dritschel, "A geenral theory for two dimensional vortex interactions," J. Fluid Mech. 293, 269-303 (1995).

${ }^{29}$ N. J. Zabusky, M. H. Hughues, and K. V. Roberts, "Contour dynamics for the euler equations in two dimensions," J. Comput. Phys. 20, 96-106 (1979).

${ }^{30}$ D. G. Dritschel, "Contour surgery: A topological reconnection scheme for extended integrations using contour dynamics," J. Comput. Phys. 77, 240-266 (1988).

${ }^{31}$ R. T. Pierrehumbert, "A family of steady, translating vortex pairs with distributed vorticity," J. Fluid Mech. 99, 129-144 (1980).

${ }^{32}$ E. A. Ryzhov and M. A. Sokolovskiy, "Interaction of a two-layer vortex pair with a submerged cylindrical obstacle in a two layer rotating fluid," Phys. Fluids 28, 056602 (2016).

${ }^{33}$ D. G. Dritschel, "The repeated filamentation of two-dimensional vorticity interfaces," J. Fluid Mech. 194, 511-547 (1988).

${ }^{34}$ L. M. Polvani and R. A. Plumb, "Rossby wave breaking, microbreaking, filamentation and secondary vortex formation: the dynamics of a perturbed vortex," J. Atmos. Sci. 49, 462-475 (1992). 